\title{
BMJ
}

\section{Exercise therapy after corticosteroid injection for moderate to severe shoulder pain: large pragmatic randomised trial}

\author{
Dickon P Crawshaw, extended scope practitioner physiotherapist,, ${ }^{1,2}$ Philip S Helliwell, senior lecturer, ${ }^{2}$ \\ Elizabeth M A Hensor, statistician, ${ }^{3}$ Elaine M Hay, professor of community rheumatology, ${ }^{4}$ Simon J Aldous, \\ extended scope physiotherapy practitioner specialising in shoulders, ${ }^{5}$ Philip G Conaghan, professor of \\ musculoskeletal medicine ${ }^{2,3}$
}

'Leeds Musculoskeletal and Rehabilitation Service, Leeds Community Healthcare, Leeds LS7 4SA

${ }^{2}$ Section of Musculoskeletal Disease, Leeds Institute of

Molecular Medicine, University of Leeds, Leeds LS7 4SA

${ }^{3} \mathrm{NIHR}$ Leeds Musculoskeletal Biomedical Research Unit, Chapel Allerton Hospital, Leeds LS7 4SA

${ }^{4}$ Arthritis Research UK National Primary Care Research Centre, Keele University, Keele, Staffordshire ST5 5BG

${ }^{5}$ Physiotherapy Department, Leeds Teaching Hospitals $N$ HS Trust, Leeds LS9 7TF Correspondence to: $\mathrm{P}$ Conaghan p.conaghan@leeds.ac.uk

Cite this as: BMJ 2010;340:c3037 doi:10.1136/bmj.c3037

\section{ABSTRACT}

Objective To compare the effectiveness of subacromial corticosteroid injection combined with timely exercise and manual therapy (injection plus exercise) or exercise and manual therapy alone (exercise only) in patients with subacromial impingement syndrome.

Design Pragmatic randomised clinical trial.

Setting Primary care based musculoskeletal service.

Patients Adults aged 40 or over with subacromial impingement syndrome with moderate or severe shoulder pain.

Interventions Injection plus exercise or exercise only. Main outcome measures Primary outcome was the difference in improvement in the total shoulder pain and disability index at 12 weeks.

Results 232 participants were randomised (115 to injection plus exercise, 117 to exercise only). The mean age was 56 (range 40-78), 127 were women, and all had had a median of 16 weeks of shoulder pain (interquartile range 12-28). At week 12 there was no significant difference between the groups in change in total pain and disability index (mean difference between change in groups 3.26 (95\% confidence interval -0.81 to 7.34 ), $\mathrm{P}=0.116)$. Improvement was significantly greater in the injection plus exercise group at week $1(6.56,4.30$ to $8.82)$ and week $6(7.37,4.34$ to 10.39$)$ for the total pain and disability index ( $\mathrm{P}$ 0.001), with no differences at week $24(-2.26,-6.77$ to $2.25, \mathrm{P}=0.324)$.

Conclusions In the treatment of patients with subacromial impingement syndrome, injection plus exercise and exercise only are similarly effective at 12 weeks.

Trial registration ISRCT 25817033; EudraCT No 2005003628-20.

\section{INTRODUCTION}

Shoulder pain is common in primary care, accounting for 11-12 per 1000 general practice consultations. ${ }^{1}$ Prevalence increases with age, peaking at around age $50 .^{2}$ Shoulder pain is often persistent, with only $50 \%$ reporting recovery after 18 months. ${ }^{3}$ Subacromial impingement syndrome (defined with Neer criteria) is reported to be the most common diagnosis. ${ }^{14-6}$
Several well designed studies in primary care have evaluated the broader concept of "shoulder pain." "7-9 Common non-operative treatments include exercise, manual therapy, and corticosteroid injections. ${ }^{10-16}$ With respect to subacromial impingement syndrome in particular, recent systematic reviews have found beneficial effects with exercise and manual therapy and with corticosteroid injections. ${ }^{1718}$ No large trials have evaluated the combination of these treatments, possibly because in traditional models of service delivery corticosteroid injections are delivered by a general practitioner and exercise or manual therapy by physio therapists. $^{7-9}$

We hypothesised that the use of exercise and manual therapy in the "window" of reduced pain after a corticosteroid injection could result in better outcomes for people with subacromial impingement syndrome. Electromyographic studies have shown that shoulder pain inhibits the rotator cuff muscles and that effective pain relief from subacromial injection of local anaesthetic can improve findings. ${ }^{19}$ As these two treatments probably work by different mechanisms, the combined treatment approach could be more effective than the single components. Using physiotherapists to deliver both treatments, we examined the short and long term effectiveness of local corticosteroid injection combined with timely exercise and manual therapy compared with exercise and manual therapy alone for people with subacromial impingement syndrome in primary care.

\section{METHODS}

We used a pragmatic approach to reflect how treatment is delivered in normal clinical practice. We recruited participants from March 2006 to August 2008. Shoulder pain was defined as pain in the shoulder region, including the upper arm, elicited or exacerbated by active or passive shoulder movement. To be included patients had to be aged 40 and older, have unilateral shoulder pain, subjectively rate their pain as moderate or severe on a 3 point scale (mild/ moderate/severe), and have a non-capsular pattern of restriction. Capsular pattern was defined as painful and 


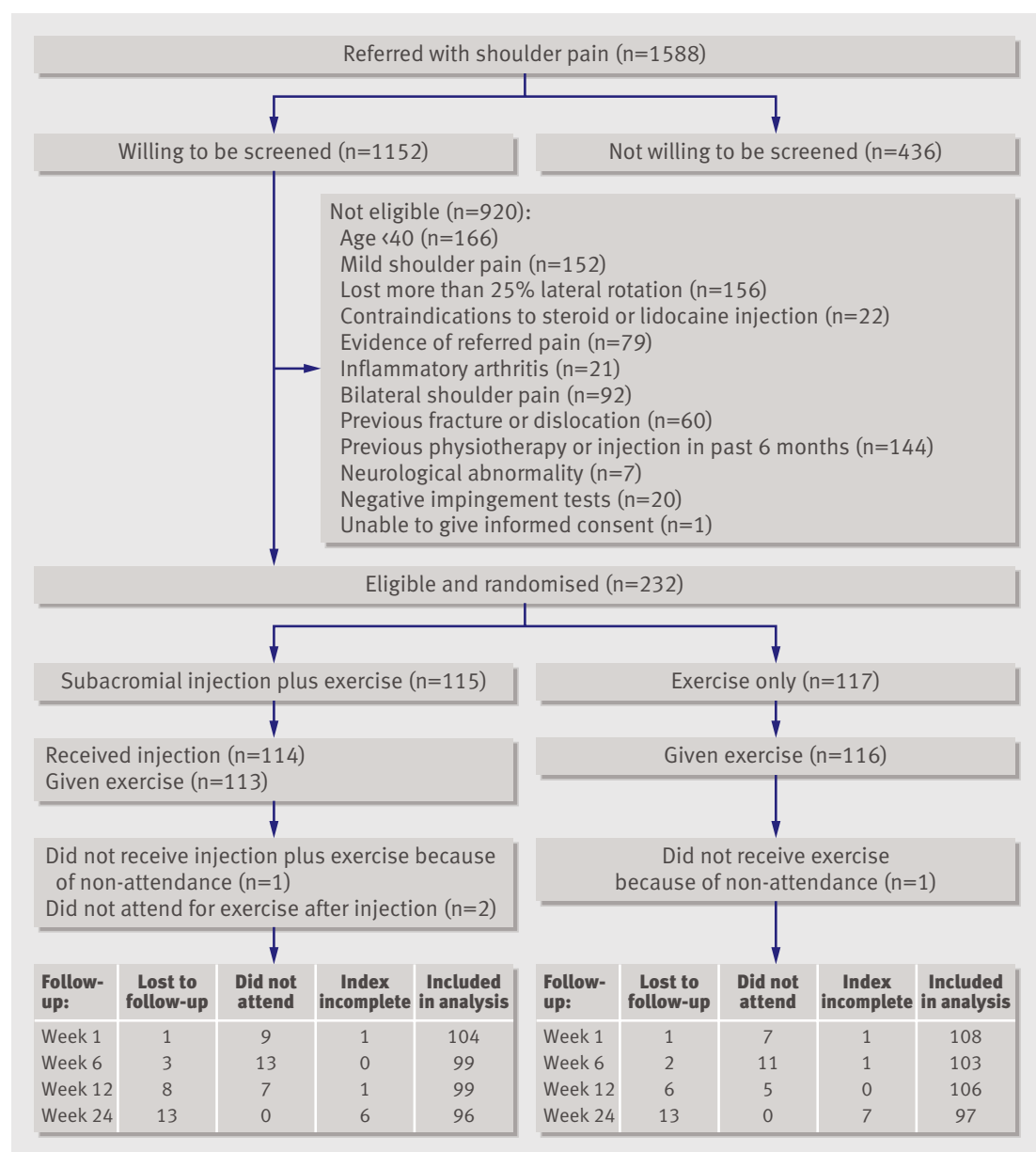

Recruitment flowchart, including reasons for non-inclusion and exclusion

limited passive glenohumeral mobility, with lateral rotation relatively more restricted than abduction and medial rotation. This pragmatic definition has been used in a previous study and is based on guidelines from the Dutch College of General Practitioners. ${ }^{8}$ Some loss of lateral rotation was permitted but no more than $25 \%$ compared with opposite side. ${ }^{20}$ Participants also had to show a Neer impingement sign (passive shoulder elevation with scapular fixed) or have positive results on the Hawkins impingement test (shoulder elevation to $90^{\circ}$, elbow flexed to $90^{\circ}$, then passively internally rotate the humerus). ${ }^{2122}$

Exclusion criteria were known blood coagulation disorders; evidence of referred pain from the cervical spine or internal organs; history of rheumatoid arthritis, polymyalgia rheumatica, or other inflammatory arthritis; bilateral shoulder pain; neurological diagnosis such as cerebrovascular event with shoulder involvement; contraindication to steroid-lidocaine injection; pregnancy or breast feeding; previous fracture, dislocation, or surgery to shoulder, upper limb, neck, or thorax; steroid injections or physiotherapy for the symptomatic shoulder within the previous six months; or inability to provide informed consent.

One author (DPC) and a research assistant recruited potentially eligible participants after screening referrals from general practitioners to the Leeds Musculoskeletal and Rehabilitation Service, a primary care based musculoskeletal service. Once consent was obtained, baseline outcome measures, including demographic variables and potential prognostic variables, were recorded. Simple block randomisation was performed for seven sites based on a computer generated randomisation list. We then randomised participants to one of the two treatment groups using an independent telephone randomisation service and booked appointments to start treatment according to allocation. While awaiting their first treatment appointment, all participants were taught a home programme of pendular exercises, which involve swinging the shoulder forwards and backwards or in a circular motion while letting the arm hang down.

\section{Interventions}

The two study arms were subacromial corticosteroid injection combined with exercise and manual therapy (referred to subsequently as injection plus exercise) or exercise and manual therapy alone (the exercise only group). DPC and SJA delivered training on the study arms to the study physiotherapists in a standardised two hour training session. The study physiotherapists were also given a trial manual to reinforce the study protocol. Participants allocated to the injection plus exercise group were injected with a lateral approach at the mid-point of the acromion with $20 \mathrm{mg}$ triamcinolone acetonide mixed with $4.5 \mathrm{ml} 1 \%$ lidocaine (lignocaine).$^{23}$ Physiotherapists with a diploma in injection therapy performed all injections under a "patient specific direction." The injection could be repeated after six weeks in patients with ongoing moderate to severe pain.

Both groups were given standard advice to avoid activities that caused or provoked pain; stop all sporting activity and training; avoid using the arm for overhead activities; and avoid repetitive movements or activities that could have contributed to the shoulder symptoms for one week.

Both groups received a programme delivered by a physiotherapist that started one week after the subacromial injection or immediately in the exercise only arm. The study coordinator, study physiotherapists, and a national opinion leader (a shoulder physiotherapy specialist) agreed the content of the physiotherapy intervention at a consensus meeting. The intervention comprised manual mobilisation techniques and exercises, selected from a range of commonly used procedures (see appendix on bmj.com). To individualise treatments, the treating physiotherapists chose mobilisation techniques and exercises for each patient from six mobilisation techniques and 23 exercises. Exercises were progressive as deemed appropriate by the treating physiotherapist. Resistive exercises were avoided for two weeks after the corticosteroid injection in line with professional guidelines. ${ }^{24}$ The treating therapist was asked to include a manual therapy technique at least once over the course of the participant's treatment. Manual therapy is the application of specific 
techniques by the therapist to mobilise joints and soft tissues. It was included as some research has shown that combined with exercise this is more effective than exercise alone. ${ }^{2526}$ The patients attended as many sessions as deemed necessary by the treating physiotherapist. We ascertained the number of participants receiving the trial interventions in the two groups from the physiotherapy records and the therapy log sheet completed by the treating physiotherapist.

\section{Outcome measures}

The primary outcome measure was the shoulder pain and disability index (SPADI) at 12 weeks. ${ }^{27}$ Scores range from 0-100 for each of two subscales representing pain and disability. A total score is obtained by taking the mean of these two subscale scores; a score of 100 indicates severe pain and disability. We included global assessment of change compared with baseline (a five point scale of "complete recovery" to "much worse" $)^{7}$ as a secondary outcome measure. Outcomes were measured by self completed questionnaires at baseline and at one, six, and 12 weeks; a staff member other than the treating physiotherapist provided the questionnaires. The one week data were collected a week after the injection (before exercise started) and hence represent the efficacy of injection alone. Information was also collected on the concomitant use of analgesics.

After 12 weeks the randomised trial finished and all patients received usual care. Follow-up by postal questionnaire at 24 weeks ascertained if improvement in the groups was maintained. The treating physiotherapist completed treatment log sheets and returned them to the study office. Information on additional treatments, referrals, and patients' satisfaction was also collected from the 24 week questionnaire and the therapy log sheets. Treating therapists were also asked to complete adverse event forms.

Sample size

Williams et al found that plus or minus 10 represented a clinically important change in the shoulder pain and disability index. ${ }^{28}$ We therefore calculated our sample

Table 1|Baseline characteristics of participants with subacromial impingement syndrome according to treatment group. Figures are numbers (percentages) unless stated otherwise

\begin{tabular}{lcc} 
& $\begin{array}{c}\text { Exercise only } \\
(\mathrm{n}=117)\end{array}$ & $\begin{array}{c}\text { Injection plus exercise } \\
(\mathrm{n}=115)\end{array}$ \\
\hline Mean (SD) age (years) & $54.9(10)$ & $57.2(10.3)$ \\
\hline Women & $67 / 117(57)$ & $60 / 115(52)$ \\
\hline Median (IQR) weeks of shoulder pain & $17(12-28)$ & $14(10-26)$ \\
\hline Started after injury & $81(69)$ & $78(68)$ \\
\hline Employed & $29(25)$ & $29(25)$ \\
\hline Diabetic & $89(76)$ & $72(63)$ \\
\hline Taken painkillers in previous 48 hours & $8(7)$ & $60(52)$ \\
\hline Mean (SD) shoulder pain and disability index: & $58(50)$ & $46.25(7.84)$ \\
\hline Total & & $50.21(8.13)$ \\
\hline Pain & $47.26(9.65)$ & $42.29(8.52)$ \\
\hline Function & $52.02(9.91)$ & \\
\hline IQR=interquartile range. & $42.49(11.05)$ & \\
\hline
\end{tabular}

size on the ability to detect a difference in treatment groups of 10 points or more in this score. Carette et al reported standard deviations around the mean change in scores from baseline to three months of 24.3 for those receiving a combination of steroid injections and physiotherapy and 24.5 for those receiving physiotherapy alone. ${ }^{20}$ Using these data, we estimated that we needed 95 patients per arm to show a significant difference at the $5 \%$ level with $80 \%$ power. With allowance for a 15\% dropout rate, we therefore required 112 patients per arm.

\section{Analysis}

Patients' data were analysed according to the randomised group irrespective of deviations from the protocol. Results are presented for patients with data available. In addition to those who were lost to follow-up, some participants for whom we had data at 24 weeks either did not attend for one or more intervening visits or did not fully complete the shoulder pain and disability index. We excluded patients with missing data at a particular time point from the analysis at that time point.

\section{Rasch transformation of pain and disability index}

To provide interval scaling, we transformed the ordinal data from the shoulder pain and disability index by Rasch analysis, with adequate model fit $\left(\chi^{2}=34.46\right.$, $\mathrm{df}=36 ; \mathrm{P}=0.542$ ) and strict unidimensionality (independent $t$ test $<5 \%) .{ }^{29}$

\section{Statistical tests}

We had three primary outcomes of interest at the primary 12 week end point: change in total score on the shoulder pain and disability index and in each of the two subscales for pain and function. Secondary outcomes included change in the three measures on the shoulder pain and disability index at each of the secondary end points, patients' global assessment of change at each end point, and subgroup analyses of patients in the exercise only group who subsequently required an injection. We analysed covariance of changes in shoulder pain and disability index measures, taking baseline values as covariates. We used Pearson's $\chi^{2}$ tests to compare patients' global assessment of change between groups and an independent Student's $t$ test to compare total shoulder pain and disability index at week 12 between patients in the exercise only group who subsequently required an injection and those who did not. Bonferroni correction for multiple comparisons set the critical significance to $\mathrm{P}<0.005$ for secondary analysis of covaraince and to $\mathrm{P}<0.0125$ for Pearson's $\chi^{2}$ tests. Analyses were carried out in SPSS 16.0.2.

\section{RESULTS}

Over 30 months we screened 1588 referrals for shoulder pain (figure). Of the 232 participants included, 115 were randomised to subacromial injection plus exercise and 117 to exercise only. Baseline characteristics were similar in both groups (table 1), though patients in the exercise only group had slightly 
Table 2 |Change in mean scores on shoulder pain and disability index over time

\begin{tabular}{|c|c|c|c|c|}
\hline & \multicolumn{2}{|c|}{ Change in mean score $(95 \% \mathrm{Cl})$} & \multirow[b]{2}{*}{ Difference $(95 \% \mathrm{Cl})$} & \multirow[b]{2}{*}{$P$ value } \\
\hline & Exercise only & Injection plus exercise & & \\
\hline \multicolumn{5}{|l|}{ Week 1} \\
\hline No of patients & 108 & 104 & - & - \\
\hline \multicolumn{5}{|l|}{ Score: } \\
\hline Total & $-1.53(-3.11$ to 0.056$)$ & $-8.08(-9.69$ to -6.47$)$ & $6.56(4.30$ to 8.82$)$ & $<0.001$ \\
\hline Pain & $-1.01(-2.68$ to -0.66$)$ & $-9.04(-10.74$ to -7.33$)$ & $8.02(5.64$ to 10.41$)$ & $<0.001$ \\
\hline Disability & $-2.01(-3.68$ to -0.34$)$ & $-7.16(-8.86$ to -5.46$)$ & $5.15(2.77$ to 7.53$)$ & $<0.001$ \\
\hline \multicolumn{5}{|l|}{ Week 6} \\
\hline No of patients & 103 & 99 & - & - \\
\hline \multicolumn{5}{|l|}{ Score: } \\
\hline Total & $-6.88(-8.99$ to -4.76$)$ & $-14.24(-16.40$ to -12.09$)$ & 7.37 (4.34 to 10.39$)$ & $<0.001$ \\
\hline Pain & $-7.29(-9.57$ to -5.02$)$ & $-15.02(-17.34$ to -12.71$)$ & 7.73 (4.48 to 10.98$)$ & $<0.001$ \\
\hline Disability & $-6.43(-8.57$ to -4.29$)$ & $-13.50(-15.68$ to -11.31$)$ & 7.07 (4.01 to 10.12$)$ & $<0.001$ \\
\hline \multicolumn{5}{|l|}{ Week 12} \\
\hline No of patients & 106 & 99 & - & - \\
\hline \multicolumn{5}{|l|}{ Score: } \\
\hline Total & $-13.09(-15.92$ to -10.26$)$ & $-16.35(-19.28$ to -13.43$)$ & $3.26(-0.81$ to 7.34$)$ & 0.116 \\
\hline Pain & $-13.29(-16.39$ to -10.19$)$ & $-17.11(-20.31$ to -13.91$)$ & $3.82(-0.65$ to 8.29$)$ & 0.093 \\
\hline Disability & $-12.83(-15.59$ to -10.06$)$ & $-15.74(-18.59$ to -12.90$)$ & $2.92(-1.05$ to 6.88$)$ & 0.149 \\
\hline \multicolumn{5}{|l|}{ Week 24} \\
\hline No of patients & 97 & 96 & - & - \\
\hline \multicolumn{5}{|l|}{ Score: } \\
\hline Total & $-17.05(-20.23$ to -13.88$)$ & $-14.79(-17.99$ to -11.60$)$ & $-2.26(-6.77$ to 2.25$)$ & 0.324 \\
\hline Pain & $-16.67(-19.94$ to -13.40$)$ & $-14.53(-17.86$ to -11.19$)$ & $-2.14(-6.83$ to 2.54$)$ & 0.368 \\
\hline Disability & $-16.87(-20.00$ to -13.74$)$ & $-14.90(-18.03$ to -11.76$)$ & $-1.98(-6.40$ to 2.45$)$ & 0.380 \\
\hline
\end{tabular}

longer duration of symptoms and were more likely to be employed. Fourteen patients (eight in injection plus exercise group; six in exercise only group) were lost to follow-up before the 12 week visit. Twelve patients (seven and five, respectively) did not attend the week 12 visit, and one patient in the injection plus exercise group did not fully complete the shoulder pain and disability index. Data from the 12 week questionnaire were therefore available for 205 (88\%). A total of 26 patients (13 in each group) did not return the 24 week questionnaire, and 13 patients (six and seven, respectively) did not fully complete the questionnaire at week 24 . Data from the 24 week questionnaire were therefore available for 193 (83\%). The 27 patients who were not included in the analysis of the primary outcomes at 12 weeks were slightly younger than those with data available (mean age 51.0 (SD 9.4) v 56.7 (SD 9.9)) but a comparable proportion were women $(15 / 27(56 \%) v$ $112 / 205(55 \%)$ and had experienced shoulder pain of similar duration on entry to the study (median 16 weeks for both), and they did not differ substantially in terms of baseline total index score (mean 46.3 (SD 4.9) v 46.8 (SD 9.2)), pain subscale score (49.9 (SD 5.7) v 51.3 (SD 9.5)), or function subscale score (42.8 (SD 30.9) v 42.3 (SD 10.3)).

\section{Compliance with protocol}

In the injection plus exercise group 114 participants received one injection, and four participants received a second injection. One participant randomised to the injection plus exercise group did not receive any treatment because of non-attendance. One participant in the exercise only group did not attend for any treatment. Six participants in the exercise only group found their pain intolerable and opted to have a steroid injection before 12 weeks. Two participants in the combination group received an injection but did not attend any physiotherapy appointments. Two hundred treatment logs were returned (98 in injection plus exercise group; 102 in exercise only group). The treatment logs indicated that all patients underwent an exercise programme. Some patients did not receive manual therapy (10/98 in injection plus exercise; 5/102 in exercise only group). The 28 remaining participants for whom we did not have a treatment log had their appointment records checked and had all attended at least one session of physiotherapy. Participants in both groups attended a median of six physiotherapy sessions.

\section{Primary outcome}

Table 2 shows the change in mean scores on the shoulder pain and disability index over time. At week 12 there was no significant difference between the groups for the pain subscale, disability subscale, or total score. Addition of duration of symptoms and employment status as covariates did not affect the results nor did adjusting for additional steroid injections required by patients in the exercise only group (data not shown).

For comparison with the Rasch transformed data, the adjusted mean change in the original total shoulder 
Table 3| Participants' global assessment of change from baseline

\begin{tabular}{|c|c|c|c|}
\hline Outcome & Exercise only & Injection plus exercise & Pvalue* \\
\hline \multicolumn{4}{|l|}{ Week 1} \\
\hline No of patients & 104 & 97 & \multirow{6}{*}{$x^{2}=24.0, P<0.001$} \\
\hline Completely recovered & 0 & $3(3)$ & \\
\hline Improved but still some problems & $50(48)$ & $72(74)$ & \\
\hline No change & $40(39)$ & $21(22)$ & \\
\hline Worse & $13(13)$ & $1(1)$ & \\
\hline Much worse & $1(1)$ & 0 & \\
\hline \multicolumn{4}{|l|}{ Week 6} \\
\hline No of patients & 100 & 94 & \multirow{6}{*}{$X^{2}=14.6, P=0.006$} \\
\hline Completely recovered & $1(1)$ & $8(9)$ & \\
\hline Improved but still some problems & $77(77)$ & $78(83)$ & \\
\hline No change & $15(15)$ & $6(6)$ & \\
\hline Worse & $7(7)$ & $1(1)$ & \\
\hline Much worse & 0 & $1(1)$ & \\
\hline \multicolumn{4}{|l|}{ Week 12} \\
\hline No of patients & 104 & 101 & \multirow{6}{*}{$X^{2}=5.4, P=0.248$} \\
\hline Completely recovered & $8(8)$ & $15(15)$ & \\
\hline Improved but still some problems & $81(76)$ & $70(71)$ & \\
\hline No change & $11(10)$ & $12(12)$ & \\
\hline Worse & $5(5)$ & $1(1)$ & \\
\hline Much worse & $1(1)$ & $1(1)$ & \\
\hline \multicolumn{4}{|l|}{ Week 24} \\
\hline No of patients & 104 & 101 & \multirow{6}{*}{$X^{2}=2.3, P=0.672$} \\
\hline Completely recovered & $17(16)$ & $16(16)$ & \\
\hline Improved but still some problems & $69(66)$ & $71(70)$ & \\
\hline No change & $10(10)$ & $11(11)$ & \\
\hline Worse & $5(5)$ & $2(2)$ & \\
\hline Much worse & 3 (3) & $1(1)$ & \\
\hline
\end{tabular}

*For change compared with baseline. recovery or improvement was similar in both groups. At 12 weeks, however, there was still a higher complete recovery rate in the injection plus exercise group than in the exercise only group (15/101 $v 8 / 104)$.

At 24 weeks the proportion still taking painkillers for their shoulder pain was higher in the exercise only group than in the injection plus exercise group (39/ $102(38 \%)$ v 28/100 (28\%)). Table 4 shows the additional treatments required in both groups. In the exercise only group 37 patients went on to have an injection after the 12 week visit compared with nine patients in the injection plus exercise group. Those in the exercise only group who had an injection had a higher mean score on the total shoulder pain and disability index at 12 weeks ( $n=37$, mean score $40.6,35.9$ to 45.4 ) than those who did not $(\mathrm{n}=69$, mean score $30.6,26.9$ to 34.3) (independent Student's $t$ test $-3.28, \mathrm{P}=0.001$ ). These patients also showed a smaller improvement from baseline $(-7.2(-11.9$ to -2.5$)$ in those who received an injection $v-16.6(-20.0$ to -13.4$)$ in those who did not; $F=10.10, \mathrm{P}=0.002)$. Most participants reported they were satisfied or very satisfied with their care over 24 weeks (91/97 (94\%) in injection plus exercise group; 93/104 (90\%) in exercise only group). No adverse events were reported in either group.

\section{DISCUSSION}

In this large pragmatic randomised trial on the management of subacromial impingement syndrome by physiotherapists we found no significant difference in the score on the shoulder pain and disability index at three months in participants who received a combination of injection and exercise compared with those who received exercise therapy alone. In agreement with two other studies investigating single treatments in the management of shoulder pain, we found outcomes at six month were similar in both groups. ${ }^{78}$ There were, however, differences between groups for our secondary outcomes. Significantly earlier improvements in pain and functional disability at one and six weeks were seen in the group given corticosteroid injection combined with exercise therapy.

\section{Results in context}

Our findings add to the current evidence base, including a head to head comparison of exercise therapy prescribed by a physiotherapist with local steroid injection delivered by a general practitioner, which found similar outcomes at six weeks and six months. ${ }^{7}$ Although group during the 24 weeks of follow-up did not affe the results (data not shown). Improvement was significantly greater in the injection plus exercise group at week 1 and 6 .

Table 3 shows participants' global assessment of overall change compared with baseline. At week 1, $50 / 104(48 \%)$ reported recovery or improvement in the exercise only group compared with 75/97 (77\%) in the injection plus exercise group. At week 6, 78/ $100(78 \%)$ reported recovery or improvement in the exercise only group compared with 86/94 (92\%) in the combined injection plus exercise group. By week 12 and 24 the percentage of participants reporting
Table $4 \mid$ Additional treatments and referrals

\begin{tabular}{|c|c|c|}
\hline Additional interventions & $\begin{array}{l}\text { Exercise only } \\
\qquad(n=117)\end{array}$ & $\begin{array}{l}\text { Injection plus } \\
\text { exercise }(n=115)\end{array}$ \\
\hline Injection before $12 / 52$ & $6(5)$ & - \\
\hline Injection after $12 / 52$ & $37(32)$ & $9(9)$ \\
\hline $\begin{array}{l}\text { Ultrasound guided } \\
\text { injection }\end{array}$ & $13(11)$ & $7(6)$ \\
\hline Ultrasound scan & $9(8)$ & $9(8)$ \\
\hline Orthopaedic referral & $9(8)$ & $8(7)$ \\
\hline
\end{tabular}




\section{WHAT IS ALREADY KNOWN ON THIS TOPIC}

Shoulder pain is common, persistent, and often caused by subacromial impingement syndrome

Exercise, manual therapy, and corticosteroid injections are common interventions in primary care for this condition

\section{WHAT THIS STUDY ADDS}

Steroid injection combined with exercise is of similar effectiveness to exercise only at 12 weeks

A third of patients treated with exercise and manual therapy alone do not improve sufficiently by 12 weeks and will opt for a steroid injection

Earlier improvement in pain and function is seen with corticosteroid injection combined with exercise and manual therapy

there was no significant difference between the groups at 12 weeks, we identified the partial improvers or nonimprovers by monitoring the additional injections that participants required once the randomised trial finished after 12 weeks, when usual care resumed. In the exercise only group there was a significantly higher week 12 score on the shoulder pain and disability index for those participants who required a subsequent injection from 12-24 weeks compared with those who were treated only with exercise therapy. Results from the current study would suggest that about a third of adults with impingement and moderate or severe shoulder pain will not respond adequately to exercise therapy alone within three months.

In examining results from recent high quality randomised controlled trials for common musculoskeletal problems, Foster et al commented on the trend for no or very small differences between the effectiveness of different approaches when based on long term outcomes (6-12 months). ${ }^{30}$

Shorter term outcomes might, of course, be more relevant to people disabled with shoulder pain and to practitioners deciding which treatment to choose in clinical practice. A recent UK trial comparing corticosteroid injection with local anaesthetic injection for rotator cuff problems also highlighted the importance of looking at early outcomes. ${ }^{31}$ It was for this reason that we included a one week outcome measure in the current study. We noted rapid improvement from the steroid injection at one week, and the combined injection and exercise protocol resulted in significantly greater improvement in pain and functional disability at six weeks. If early pain relief is a priority, then adding local steroid injection to a course of physiotherapy would seem to be the best option for patients.

In our trial all therapies were delivered by physiotherapists working in a primary care based musculoskeletal service, and no extra NHS resources were required to implement the study interventions. In routine clinical practice in the UK, combining these treatments in a timely fashion could be problematic for practical reasons related to the skills of practitioners and service issues such as waiting lists for physiotherapy. In this study physiotherapists provided both arms of the study; in other large trials different professions have provided the treatment arms. ${ }^{7-931}$

The need to avoid selection of a heterogeneous group in research into shoulder pain has been highlighted. ${ }^{32}$ Although clinical classification is difficult, we aimed to exclude conditions for which the study protocol was not appropriate, such as adhesive capsulitis and inflammatory arthritis, and patients with mild shoulder pain and those aged under 40 years. Severe shoulder pain at initial presentation has been shown to be a prognostic indicator for persistent symptoms. ${ }^{334}$ In keeping with two previous studies that excluded patients with low scores on the shoulder pain and disability index ${ }^{2035}$ we deliberately included only patients who rated their pain as moderate or severe because patients with mild pain would not routinely be offered an injection in clinical practice. Of those patients not eligible for the study, $152(17 \%)$ reported mild shoulder pain. Other studies investigating corticosteroid injections have not used the level of pain or degree of functional disability as specific entry criteria. ${ }^{36-41}$ We excluded patients aged 40 and under to avoid including younger patients with subacromial impingement related to a sporting injury, who are not usually offered steroid injections as an initial treatment, which was the consensus view of the physiotherapists who designed the intervention package. A recent study indicated that nearly $80 \%$ of shoulder pain is found in patients aged 40 and over ${ }^{2}$; our study therefore reflecting the age group with the highest prevalence of shoulder pain.

\section{Limitations of study}

Because of this study's pragmatic design, participants were not blinded to their interventions and there could have been a placebo or non-specific effect caused by the injection. We therefore accept that the total treatment response probably includes both treatment and associated placebo effects, as is the case in routine clinical practice. ${ }^{42}$ We did not set out to examine which component of the non-pharmacological intervention (exercise or manual therapy, or both) is effective. Although evidence is emerging about the efficacy of certain interventions-for example, exercise combined with manual therapy seems more effective than exercise alone for subacromial impingement syndrome,${ }^{2526}$ and ultrasound guided injections might have better outcomes than "blind" injections ${ }^{43}$ - questions about the optimal use of injection and physiotherapy interventions remain and continue to be important for future research.

We thank the participating physiotherapists (L Ackerman, D Aldous, S Banerjee, R Batley, R Bodey, F Brown, N Clay, K Charman, C Comer, J Cousins, S Dadswell, V Doemling, J Flint, S Foster, J Glover, N Henderson, A Ibison, V Johnstone, E Jones, N Jones, T Langford, I Muirhead, E Newton, R Osborn, M Oxley, R Partner, J Patterson, G Reader-Peate, A Reilly, J Smith, S Smith, J Sneath, A Thompson, K Thompson, B Urquhart, S Urquhart, S Walby, D Walker, A White), the management and administration staff at Leeds Musculoskeletal and Rehabilitation Service (L Berry, W Brocklehurst, T Butterfield, C Cagna, A Griffiths, V Hughes, S Johnson, D Lambert, C McNamara, J Moody, J Rogers, S Strickland), the research assistant ( $K$ Lewis), and the patients who made the trial possible. We also thank J Klaber Moffett for her advice on the original 
study proposal and the Institute of Rehabilitation, University of Hull, for providing the telephone randomisation service.

Contributors: All authors participated in the planning of the study and contributed to the protocol. EMAH analysed the data. DPC drafted the manuscript, which was revised by the other authors. PGC is guarantor Funding: The study was funded by a project grant 17236 from Arthritis Research UK.

Competing interests: All authors have completed the Unified Competing Interest form at www.icmje.org/coi_disclosure.pdf (available on request from the corresponding author) and declare that (1) DPC and PGC have support from Arthritis Research UK for the submitted work. All authors declare no interests under (2), (3), and (4).

Ethical approval: This study was approved by the local research ethics committee, and informed consent was given by all patients. Data sharing: The dataset will be available from the corresponding author as part of an academic collaboration.

1 Van der Windt DA, Koes BW, de Jong BA, Bouter LM. Shoulder disorders in general practice: incidence, patient characteristics, and management. Ann Rheum Dis 1995;54:959-64.

2 Linsell L, Dawson J, Zondervan K, Rose P, Randall T, Fitzpatrick R, et al. Prevalence and incidence of adults consulting for shoulder conditions in UK primary care; patterns of diagnosis and referral. Rheumatology (Oxford) 2006;45:215-21.

3 Croft P, Pope D, Silman A. The clinical course of shoulder pain: prospective cohort study in primary care. Primary Care Rheumatology Society Shoulder Study Group. BMJ 1996;313:601-2.

4 Neer CS 2nd. Impingement lesions. Clin Orthop Relat Res 1983;173:70-7.

5 Ostor AJ, Richards CA, Prevost AT, Speed CA, Hazleman BL. Diagnosis and relation to general health of shoulder disorders presenting to primary care. Rheumatology (Oxford) 2005;44:800-5.

6 Chard MD, Hazleman R, Hazleman BL, King RH, Reiss BB. Shoulder disorders in the elderly: a community survey. Arthritis Rheum 1991;34:766-9.

7 Hay EM, Thomas E, Paterson SM, Dziedzic K, Croft PR. A pragmatic randomised controlled trial of local corticosteroid injection and physiotherapy for the treatment of new episodes of unilateral shoulder pain in primary care. Ann Rheum Dis 2003;62:394-9.

8 Van der Windt DA, Koes BW, Deville W, Boeke Al, de Jong BA, Bouter LM. Effectiveness of corticosteroid injections versus physiotherapy for treatment of painful stiff shoulder in primary care: randomised trial. BMJ 1998;317:1292-6.

9 Winters IC, Sobel JS, Groenier KH, Arendzen HJ, Meyboom-de Jong B. Comparison of physiotherapy, manipulation, and corticosteroid injection for treating shoulder complaints in general practice: randomised, single blind study. BMJ 1997;314:1320-5.

10 Green S, Buchbinder R, Glazier R, Forbes A. Systematic review of randomised controlled trials of interventions for painful shoulder: selection criteria, outcome assessment, and efficacy. BMJ 1998;316:354-60

11 Green S, Buchbinder R, Hetrick S. Physiotherapy interventions for shoulder pain. Cochrane Database Syst Rev 2003;2:CD004258.

12 Sauers EL. Effectiveness of rehabilitation for patients with subacromial impingement syndrome. J Athl Train 2005;40:221-3.

13 Morrison DS, Frogameni AD, Woodworth P. Non-operative treatment of subacromial impingement syndrome. J Bone Joint Surg Am 1997;79:732-7.

14 Engebretsen K, Grotle M, Bautz-Holter E, Sandvik L, Juel NG, Ekeberg OM, et al. Radial extracorporeal shockwave treatment compared with supervised exercises in patients with subacromial pain syndrome: single blind randomised study. $B M J$ 2009;339:b3360.

15 Brox II, Staff PH, Ljunggren AE, Brevik JI. Arthroscopic surgery compared with supervised exercises in patients with rotator cuff disease (stage II impingement syndrome). BMJ 1993;307:899-903.

16 Brox JI, Gjengedal E, Uppheim G, Bohmer AS, Brevik JI, Ljunggren AE, et al. Arthroscopic surgery versus supervised exercises in patients with rotator cuff disease (stage II impingement syndrome): a prospective, randomized, controlled study in 125 patients with a 2 1/2-year follow-up. / Shoulder Elbow Surg 1999;8:102-11.

17 Kuhn JE. Exercise in the treatment of rotator cuff impingement: a systematic review and a synthesized evidence-based rehabilitation protocol. J Shoulder Elbow Surg 2009;18:138-60.

18 Arroll B, Goodyear-Smith F. Corticosteroid injections for painful shoulder: a meta-analysis. BrJ Gen Pract 2005;55:224-8.

19 Brox II, Roe C, Saugen E, Vollestad NK. Isometric abduction muscle activation in patients with rotator tendinosis of the shoulder. Arch Phys Med Rehabil 1997;78:1260-7.
20 Carette S, Moffet H, Tardif J, Bessette L, Morin F, Fremont P, et al. Intraarticular corticosteroids, supervised physiotherapy, or a combination of the two in the treatment of adhesive capsulitis of the shoulder: a placebo-controlled trial. Arthritis Rheum 2003;48:829-38.

21 Calis M, Akgun K, Birtane M, Karacan I, Calis H, Tuzun F. Diagnostic values of clinical diagnostic tests in subacromial impingement syndrome. Ann Rheum Dis 2000;59:44-7.

22 MacDonald PB, Clark P, Sutherland K. An analysis of the diagnostic accuracy of the Hawkins and Neer subacromial impingement signs. I Shoulder Elbow Surg 2000;9:299-301.

23 Saunders S, Cameron G. Injection techniques in orthopaedic and sports medicine. W B Saunders, 1997.

24 ACPOM. A clinical guideline for the use of injection therapy by physiotherapists. Chartered Society of Physiotherapy, 1999.

25 Bang MD, Deyle GD. Comparison of supervised exercise with and without manual physical therapy for patients with shoulder impingement syndrome. J Orthop Sports Phys Ther 2000;30:126-37.

26 Senbursa G, Baltaci G, Atay A. Comparison of conservative treatment with and without manual physical therapy for patients with shoulder impingement syndrome: a prospective, randomized clinical trial. Knee Surg Sports Traumatol Arthrosc 2007;15:915-21.

27 Roach KE, Budiman-Mak E, Songsiridej N, Lertratanakul Y. Development of a shoulder pain and disability index. Arthritis Care Res 1991;4:143-9.

28 Williams JW Jr, Holleman DR Jr, Simel DL. Measuring shoulder function with the shoulder pain and disability index. J Rheumatol 1995;22:727-32.

29 Tennant A, Conaghan PG. The Rasch measurement model in rheumatology: what is it and why use it? When should it be applied, and what should one look for in a Rasch paper? Arthritis Rheum 2007;57:1358-62.

30 Foster NE, Dziedzic KS, van der Windt DA, Fritz IM, Hay EM. Research priorities for non-pharmacological therapies for common musculoskeletal problems: nationally and internationally agreed recommendations. BMC Musculoskelet Disord 2009;10:3.

31 Watson J, Helliwell P, Morton V, Adebajo A, Dickson J, Russell I, et al. Shoulder acute pain in primary healthcare: is retraining effective for GP principals? SAPPHIRE-a randomized controlled trial. Rheumatology (Oxford) 2008;47:1795-802.

32 Bamii AN. Treatment of shoulder pain. Ann Rheum Dis 2004;63:607.

33 Van der Windt DA, Koes BW, Boeke AJ, Deville W, De Jong BA, Bouter LM. Shoulder disorders in general practice: prognostic indicators of outcome. Br / Gen Pract 1996;46:519-23.

34 Kuijpers T, van der Windt DA, Boeke AJ, Twisk JW, Vergouwe Y, Bouter LM, et al. Clinical prediction rules for the prognosis of shoulder pain in general practice. Pain 2006;120:276-85.

35 Ekeberg OM, Bautz-Holter E, Tveita EK, Juel NG, Kvalheim S, Brox Jl. Subacromial ultrasound guided or systemic steroid injection for rotator cuff disease: randomised double blind study. BMJ 2009;338:a3112.

36 Adebajo AO, Nash P, Hazleman BL. A prospective double blind dummy placebo controlled study comparing triamcinolone hexacetonide injection with oral diclofenac $50 \mathrm{mg}$ TDS in patients with rotator cuff tendinitis. J Rheumatol 1990;17:1207-10.

37 Petri M, Dobrow R, Neiman R, Whiting-O'Keefe Q, Seaman WE. Randomized, double-blind, placebo-controlled study of the treatment of the painful shoulder. Arthritis Rheum 1987;30:1040-5.

38 Vecchio PC, Hazleman BL, King RH. A double-blind trial comparing subacromial methylprednisolone and lignocaine in acute rotator cuff tendinitis. Br I Rheumatol 1993;32:743-5.

39 Blair B, Rokito AS, Cuomo F, Jarolem K, Zuckerman JD. Efficacy of injections of corticosteroids for subacromial impingement syndrome. / Bone Joint Surg Am 1996;78:1685-9.

40 McInerney J), Dias J, Durham S, Evans A. Randomised controlled trial of single, subacromial injection of methylprednisolone in patients with persistent, post-traumatic impingement of the shoulder. Emerg Med / 2003;20:218-21.

41 Plafki C, Steffen R, Willburger RE, Wittenberg RH. Local anaesthetic injection with and without corticosteroids for subacromial impingement syndrome. Int Orthop 2000;24:40-2.

42 Roland M, Torgerson DJ. What are pragmatic trials? BMJ 1998;316:285.

43 Naredo E, Cabero F, Beneyto P, Cruz A, Mondejar B, Uson J, et al. A randomized comparative study of short term response to blind injection versus sonographic-guided injection of local corticosteroids in patients with painful shoulder. / Rheumatol 2004:31:308-14

Accepted: 8 April 2010 